\title{
DECOUPLED ENERGY STABLE SCHEMES FOR PHASE-FIELD MODELS OF TWO-PHASE COMPLEX FLUIDS*
}

\author{
JIE SHEN ${ }^{\dagger}$ AND XIAOFENG YANG ${ }^{\ddagger}$
}

\begin{abstract}
We consider in this paper numerical approximations of phase-field models for twophase complex fluids. We first reformulate the phase-field models derived from an energetic variational formulation into a form which is suitable for numerical approximation and establish their energy laws. Then, we construct two classes, stabilized and convex-splitting, of decoupled time discretization schemes for the coupled nonlinear systems. These schemes are unconditionally energy stable and lead to decoupled elliptic equations to solve at each time step. Furthermore, these elliptic equations are linear for the stabilized version. Stability analysis and ample numerical simulations are presented.
\end{abstract}

Key words. phase-field, liquid crystals, multiphase flows, Navier-Stokes, Allen-Cahn, CahnHilliard, stability

AMS subject classifications. 65M12, 65M70, 65Z05

DOI. $10.1137 / 130921593$

1. Introduction. Complex fluids have great practical utility since the microstructure can be manipulated to produce useful mechanical, optical, or thermal properties [4,23]. Numerical studies of multiphase complex fluids is a rich area for research with important applications that can be addressed. Some numerical difficulties are (i) the moving interfaces between various components; (ii) open issues regarding wellposedness of many multiphase models leading to confusion as to whether numerical pathologies are due to the model or the methods; and most important, (iii) nonlinear coupling (hydrodynamics, interface, and microstructure) makes it very difficult to design easy-to-implement, energy stable numerical schemes.

For the first two issues above, the diffuse-interface/phase-field models, whose origin can be traced back to [38] and [46], have been proved efficient with much success. A particular advantage of the phase-field approach is that models can often be derived from an energy-based variational formalism (energetic variational approaches), leading to well-posed nonlinear coupled systems that satisfy thermodynamics-consistent energy dissipation laws. This makes it possible to carry out mathematical analysis and design numerical schemes which satisfy a corresponding discrete energy dissipation law. In recent years, the phase-field method has become one of the major tools to study a variety of interfacial phenomena (cf. [17, 2, 36, 19, 32, 49] and recent review papers $[44,22]$ and the references therein).

For phase-field models which satisfy a dissipative energy law, it is especially desirable to design numerical schemes that preserve the energy dissipation law at the

*Submitted to the journal's Computational Methods in Science and Engineering section May 20, 2013; accepted for publication (in revised form) October 21, 2013; published electronically February 27, 2014.

http://www.siam.org/journals/sisc/36-1/92159.html

†School of Mathematics and Science and Fujian Provincial Key Laboratory on Mathematical Modeling and High Performance Computing, Xiamen University, Xiamen 361005, China, and Department of Mathematics Purdue University, West Lafayette, IN 47907, USA (shen@math.purdue.edu). This author's work was partially supported by NSFC grants 91130002 and 11371298.

${ }^{\ddagger}$ Corresponding author. Department of Mathematics, University of South Carolina, Columbia, SC 29208 (xfyang@math.sc.edu). This author's work was partially supported by NSF-1200487, AFOSR FA9550-12-1-0178, and SC Epscor Gear, Gear-CI fund. 
discrete level. In particular, the dynamics of the coarse-graining (macroscopic) process described by the Allen-Cahn and Cahn-Hilliard equations may undergo rapid changes near the interface, so the noncompliance of energy dissipation laws may lead to spurious numerical solutions if the grid and time step sizes are not carefully controlled $[30,18]$. Thus, from the numerical point of view, we are particularly interested in designing simple and efficient but energy stable numerical schemes satisfying discrete energy dissipations laws.

The nematic liquid crystal is a typical complex fluid that has attracted much attention in the physical and mathematical communities (cf. [24, 25, 13, 26, 9, 27, 28] and the references therein). We consider in this paper a mixture of nematic liquid crystals and a Newtonian viscous fluid. Both phases are assumed to be incompressible and immiscible. We recall that a phase-field model to describe this mixture was derived in [49] using an energetic variational formulation. However, to simplify the computation, some nonessential nonlinear interaction terms were dropped there. While dropping these terms does simplify the implementation, it also destroys the variational structure so the phase-field system does not satisfy an energy law.

Recently in [48], we derived the phase-field model for this mixture which satisfies a dissipative energy law by keeping all nonlinear interaction terms from the energetic variational formalism. We presented numerical simulations of a nematic liquid crystal drop or filament immersed in a Newtonian viscous fluid and observed a very interesting "beads-on-a-string" interfacial morphology that is qualitative consistency with the experimental results in [3]. However, due to the explicit treatment of all nonlinear terms, the numerical scheme used in [48] does not satisfy the energy dissipation law even though it is very easy to implement. A sufficiently small time step, which can be very costly in large-scale computations, has to be used to ensure stability. Thus the main purpose of this paper is to construct a time discretization scheme which (a) is unconditionally stable, (b) satisfies a discrete energy law, and (c) leads to decoupled elliptic equations to solve at each time step. This is by no means an easy task due to highly nonlinear couplings between velocity, pressure, phase function, and director field.

The rest of the paper is organized as follows. In the next section, we describe a phase-field model for the mixture of a nematic liquid crystal and a Newtonian viscous fluid, and we derive the associated energy law. In section 3, we construct decoupled, energy stable numerical schemes for solving the coupled nonlinear system. In section 4, we present some numerical results to illustrate the efficiency of the proposed scheme and summarize our contributions.

2. The phase-field model and its energy law. We consider a mixture of nematic liquid crystals and a Newtonian incompressible viscous fluid. We use a phase function $\phi$ to identify the two fluids, namely,

$$
\phi(x, t)=\left\{\begin{aligned}
1 & \text { liquid crystal } \\
-1 & \text { viscous fluid }
\end{aligned}\right.
$$

with a thin smooth transition layer of thickness $\varepsilon$ connecting the two fluids, so the interface of the mixture is described by the zero level set $\Gamma_{t}=\{x: \phi(x, t)=0\}$.

We recall below the essential ingredients in the derivation of the phase-field model using an energetic variational procedure (cf. [49, 48]).

Let $F(\phi)=\frac{1}{4 \varepsilon^{2}}\left(\phi^{2}-1\right)^{2}$ be the usual Ginzburg-Landau double-well potential, and define the mixing energy functional 


$$
E_{\text {mix }}=\int_{\Omega} W_{\text {mix }}(\phi, \nabla \phi) d \mathbf{x}=\int_{\Omega} \lambda\left(\frac{1}{2}|\nabla \phi|^{2}+F(\phi)\right) d \mathbf{x},
$$

where $\lambda$ is the mixing energy density which can be related to the traditional surface tension density [49]. We assume that the elastic energy is isotropic, so the bulk energy can be described by the standard Oseen-Frank distortional energy [12, 24, 25]:

$$
W_{b u l k}(\mathbf{d}, \nabla \mathbf{d})=K\left(\frac{|\nabla \mathbf{d}|^{2}}{2}+G(\mathbf{d})\right),
$$

where $K$ is the elastic constant and $G(\mathbf{d})=\frac{1}{4 \eta^{2}}\left(|\mathbf{d}|^{2}-1\right)^{2}$ is a Ginzburg-Landau type penalty term, where $\eta$ is a penalization parameter, introduced to approximate the unit length constraint of $\mathbf{d}[26,27]$.

Nematic liquid crystals prefer some particular orientations along the interface between the two fluids, called the easy direction. There are two common types of anchoring conditions: planar (or parallel) anchoring, where all directions in the plane of the interface are easy directions, and homeotropic (or normal) anchoring, where the easy direction is the normal to the interface [20,9]. Namely, the anchoring energy density is given by

$$
W_{\text {anch }}(\phi, \nabla \phi, \mathbf{d}, \nabla \mathbf{d})= \begin{cases}\frac{A}{2}|\mathbf{d} \cdot \nabla \phi|^{2} & \text { parallel, } \\ \frac{A}{2}\left(|\mathbf{d}|^{2}|\nabla \phi|^{2}-|\mathbf{d} \cdot \nabla \phi|^{2}\right) & \text { homeotropic, }\end{cases}
$$

where $A(x)$ is some prescribed molecular anchoring function defining the anchoring energy.

The total energy of the hydrodynamic system is a sum of the kinetic energy $E_{k i n}$, the mixing energy $E_{m i x}$, the bulk energy $E_{b u l k}$, and the anchoring energy $E_{\text {anch }}$ [49]:

$$
\begin{aligned}
\mathscr{E}_{\text {tot }} & =E_{\text {kin }}+E_{\text {mix }}+\left(\frac{1+\phi}{2}\right)^{2} E_{\text {bulk }}+E_{\text {anch }}, \\
& =\int_{\Omega}\left(\frac{1}{2} \rho|\mathbf{u}|^{2}+W_{\text {mix }}+\left(\frac{1+\phi}{2}\right)^{2} W_{\text {bulk }}+W_{\text {anch }}\right) d \mathbf{x},
\end{aligned}
$$

where $\rho$ is the density, $\mathbf{u}$ is the fluid velocity field, and the factor $\left(\frac{1+\phi}{2}\right)^{2}$ represents the volume fraction of the nematic liquid crystal component that vanishes in the viscous liquid phase $(\phi=-1)$.

Assuming a generalized Fick's law that the mass flux be proportional to the gradient of the chemical potential $[7,15,34,32]$, we can derive the following system:

$$
\begin{aligned}
\mathbf{d}_{t}+(\mathbf{u} \cdot \nabla) \mathbf{d} & =-M_{1} \frac{\delta \mathscr{E}_{t o t}}{\delta \mathbf{d}}, \\
\phi_{t}+(\mathbf{u} \cdot \nabla) \phi & =-M_{2} \frac{\delta \mathscr{E}_{t o t}}{\delta \phi}, \\
\rho\left(\mathbf{u}_{t}+(\mathbf{u} \cdot \nabla) \mathbf{u}\right)+\nabla p & =\nabla \cdot\left(\sigma_{v i s}+\sigma_{\text {mix }}^{e}+\sigma_{\text {bulk }}^{e}+\sigma_{\text {anch }}^{e}\right), \\
\nabla \cdot \mathbf{u} & =0,
\end{aligned}
$$

where $p$ is the pressure, $M_{1}$ is the relaxation parameter of liquid crystals, $M_{2}$ is the mobility parameter of the phase function, $\sigma_{v i s}=\nu\left(\frac{\nabla \mathbf{u}+(\nabla \mathbf{u})^{T}}{2}\right)$ is the usual Newtonian viscous stress, and $\sigma_{m i x}^{e}, \sigma_{b u l k}^{e}, \sigma_{\text {anch }}^{e}$ are the induced elastic stress by each energy 
component which can be obtained by the least action principle [28, 29, 32, 49]. The variational derivative $\frac{\delta \mathscr{E}_{\text {tot }}}{\delta \phi}$ can be taken in $H^{-1}$, leading to the (conserved) CahnHilliard phase equation,

$$
\begin{aligned}
\phi_{t}+(\mathbf{u} \cdot \nabla) \phi & =-M_{2} \Delta \mu, \\
\mu & =\lambda(\Delta \phi-f(\phi))-K\left(\frac{1+\phi}{2}\right)\left(\frac{|\nabla \mathbf{d}|^{2}}{2}+G(\mathbf{d})\right)-\frac{\delta E_{\text {anch }}}{\delta \phi},
\end{aligned}
$$

where $f(\phi)=F^{\prime}(\phi)$, or in $L^{2}$, leading to the (nonconserved) Allen-Cahn phase equation,

$$
\phi_{t}+(\mathbf{u} \cdot \nabla) \phi=M_{2} \mu
$$

where $\mu$ is given in (2.10).

Remark 2.1. We note that solutions of the (conserved) Cahn-Hilliard phase equation, with suitable boundary conditions, satisfy the desired conservation property, $\partial_{t} \int_{\Omega} \phi d x=0$, which is not satisfied by the solutions of the (nonconserved) Allen-Cahn equation. However, one can add a scalar Lagrange multiplier in (2.11) to enforce this conservation property (cf. [47]). Since the Lagrange multiplier does not introduce any mathematical or numerical difficulty, we shall not include it in the discussion below.

Remark 2.2. For simplicity, we consider in this paper the cases where the two fluids have matching density and viscosity, i.e., $\rho_{1}=\rho_{2}$ and $\nu_{1}=\nu_{2}$. When the two fluids have different densities with a relative small density ratio, one can use the so-called Boussinesq approximation which models the effect of different densities by a gravitational force $[32,47]$. The case of difference viscosities can usually be dealt with in a straightforward manner by assuming the viscosity is a linear or harmonic average of the phase function.

Let $\rho_{1}=\rho_{2}=1$ and $\nu_{1}=\nu_{2}=\nu$. The system (2.6)-(2.9), with the Allen-Cahn phase equation, becomes

$$
\phi_{t}+(\mathbf{u} \cdot \nabla) \phi=M_{2} \lambda(\Delta \phi-f(\phi))-M_{2} K\left(\frac{1+\phi}{2}\right)\left(\frac{|\nabla \mathbf{d}|^{2}}{2}+G(\mathbf{d})\right)-M_{2} A_{\mathscr{A}_{\phi}},
$$

$\mathbf{u}_{t}+(\mathbf{u} \cdot \nabla) \mathbf{u}-\nu \Delta \mathbf{u}+\nabla p+\nabla \cdot\left(\lambda \nabla \phi \otimes \nabla \phi+K\left(\frac{1+\phi}{2}\right)^{2} \nabla \mathbf{d} \otimes \nabla \mathbf{d}+A \mathscr{A}_{\mathbf{u}}\right)=0$,

$$
\nabla \cdot \mathbf{u}=0
$$

where $f(\phi)=F^{\prime}(\phi), g(\mathbf{d})=\nabla G(\mathbf{d})$, and

$$
\begin{aligned}
& \mathscr{A}_{\phi}= \begin{cases}\nabla \cdot((\nabla \phi \cdot \mathbf{d}) \mathbf{d}) & \text { parallel, } \\
\nabla \cdot\left(|\mathbf{d}|^{2} \nabla \phi-(\nabla \phi \cdot \mathbf{d}) \mathbf{d}\right) & \text { homeotropic, }\end{cases} \\
& \mathscr{A}_{\mathbf{d}}= \begin{cases}(\mathbf{d} \cdot \nabla \phi) \nabla \phi & \text { parallel, } \\
(\nabla \phi \cdot \nabla \phi) \mathbf{d}-(\mathbf{d} \cdot \nabla \phi) \nabla \phi & \text { homeotropic, }\end{cases} \\
& \mathscr{A}_{\mathbf{u}}= \begin{cases}(\mathbf{d} \cdot \nabla \phi) \mathbf{d} \otimes \nabla \phi & \text { parallel, } \\
((\mathbf{d} \cdot \mathbf{d}) \nabla \phi-(\mathbf{d} \cdot \nabla \phi) \mathbf{d}) \otimes \nabla \phi & \text { homeotropic. }\end{cases}
\end{aligned}
$$


On the other hand, the model with the Cahn-Hilliard phase equation is obtained by replacing (2.13) in the above by (2.10).

Throughout the paper, we assume the boundary conditions

$$
\left.\mathbf{u}\right|_{\partial \Omega}=0,\left.\quad \partial_{n} \phi\right|_{\partial \Omega}=0,\left.\quad \partial_{n} \mu\right|_{\partial \Omega}=0,\left.\quad \partial_{n} \mathbf{d}\right|_{\partial \Omega}=0
$$

although all results are valid for periodic boundary conditions as well.

Since the above system was derived from an energetic variational formulation without neglecting any terms, it can be readily established that the total energy of the system (2.13)-(2.15) is dissipative. More precisely, by taking the inner product of $(2.12)$ with $\frac{\delta \mathscr{E}_{\text {tot }}}{\delta \mathbf{d}},(2.13)$ with $\frac{\delta \mathscr{E}_{\text {tot }}}{\delta \phi}$, and (2.14) with $\mathbf{u}$, and then summing up these equalities, we obtain the following energy dissipation law:

$$
\partial_{t} \mathscr{E}_{t o t}=-\int_{\Omega}\left(\nu|\nabla \mathbf{u}|^{2}+M_{1}\left|\frac{\delta \mathscr{E}_{t o t}}{\delta \phi}\right|^{2}+M_{2}\left|\frac{\delta \mathscr{E}_{t o t}}{\delta \mathbf{d}}\right|^{2}\right) d \mathbf{x}
$$

Similarly, the corresponding energy law with the Cahn-Hilliard phase equation is

$$
\partial_{t} \mathscr{E}_{t o t}=-\int_{\Omega}\left(\nu|\nabla \mathbf{u}|^{2}+M_{1}\left|\nabla \frac{\delta \mathscr{E}_{t o t}}{\delta \phi}\right|^{2}+M_{2}\left|\frac{\delta \mathscr{E}_{t o t}}{\delta \mathbf{d}}\right|^{2}\right) d \mathbf{x}
$$

The above energy laws will enable us to prove the existence and uniqueness of the weak solution with certain smoothness by a standard Galerkin procedure [10].

3. Alternative formulations and their decoupled time discretizations. The coupled nonlinear system (2.12)-(2.19) presents formidable challenges for algorithm design and implementation, as well as numerical analysis. Although many numerical schemes perform well in practice for such time-dependent diffusion-convection dynamical system, the question of their stability remains open. It has been shown that some spurious solutions may occur if a numerical scheme does not follow the discrete energy dissipation law when the spatial grid and time step sizes are not carefully chosen (cf. [30,31, 18]). Thus the compliance of discrete energy dissipation laws usually serves as the justification of numerical schemes and results, when no benchmark solutions are available. In addition, use of unconditionally energy stable schemes enable the use of relatively large time steps, the size of which is dictated only by accuracy considerations.

The emphasis of our algorithm development is placed on designing numerical schemes that not only are easy to implement but also satisfy a discrete energy dissipation law. We will design schemes that in particular can overcome the following difficulties:

- the coupling of the velocity and pressure through the incompressible condition;

- the stiffness in the phase equation and the director equation associated with the interfacial width $\varepsilon$ and the penalty parameter $\eta$;

- the nonlinear couplings among the fluid equation, the phase equation, and the director equation.

3.1. An alternative derivation of the energy dissipation law. It is straightforward to derive the energy law (2.20) by taking the inner product of (2.12) with 
$\frac{\delta \mathscr{E}_{t o t}}{\delta \mathrm{d}},(2.13)$ with $\frac{\delta \mathscr{E}_{t o t}}{\delta \phi}$, and (2.14) with u. However, the nonlinear terms in $\frac{\delta \mathscr{E}_{\text {tot }}}{\delta \phi}$ and $\frac{\delta \mathscr{E}_{\text {tot }}}{\delta \mathrm{d}}$ involve second-order derivatives, and it is not convenient to use them as test functions in numerical approximations, making it difficult to prove the discrete energy dissipation law.

To overcome this difficulty, we first reformulate the system (2.12)-(2.19) in an alternative form which is convenient for numerical approximation. For simplicity, we shall omit the anchoring energy in this section since it does not involve any small parameter, so it is relatively safe to treat it explicitly.

By direct calculation, we have the following two identities:

$$
\begin{aligned}
\nabla \cdot\left(\left(\frac{1+\phi}{2}\right)^{2} \nabla \mathbf{d} \otimes \nabla \mathbf{d}\right)= & \left(\nabla \cdot\left(\frac{1+\phi}{2}\right)^{2} \nabla \mathbf{d}-\left(\frac{1+\phi}{2}\right)^{2} g(\mathbf{d})\right) \nabla \mathbf{d} \\
& +\nabla\left(\left(\frac{1+\phi}{2}\right)^{2}\left(\frac{|\nabla \mathbf{d}|^{2}}{2}+G(\mathbf{d})\right)\right) \\
& -\left(\frac{1+\phi}{2}\right)\left(\frac{|\nabla \mathbf{d}|^{2}}{2}+G(\mathbf{d})\right) \nabla \phi \\
\nabla \cdot(\nabla \phi \otimes \nabla \phi)= & (\Delta \phi-f(\phi)) \nabla \phi+\nabla\left(\frac{|\nabla \phi|^{2}}{2}+F(\phi)\right) .
\end{aligned}
$$

Taking the summation of the above two identities, we derive

$$
\begin{aligned}
& \lambda \nabla \cdot(\nabla \phi \otimes \nabla \phi)+K \nabla \cdot\left(\left(\frac{1+\phi}{2}\right)^{2} \nabla \mathbf{d} \otimes \nabla \mathbf{d}\right) \\
& \quad=\frac{\dot{\mathbf{d}}}{M_{1}} \nabla \mathbf{d}+\frac{\dot{\phi}}{M_{2}} \nabla \phi+\nabla\left(\lambda\left(\frac{|\nabla \phi|^{2}}{2}+F(\phi)\right)+K\left(\frac{1+\phi}{2}\right)^{2}\left(\frac{|\nabla \mathbf{d}|^{2}}{2}+G(\mathbf{d})\right)\right),
\end{aligned}
$$

where we have denoted

$$
\dot{\phi}=\phi_{t}+(\mathbf{u} \cdot \nabla) \phi, \dot{\mathbf{d}}=d_{t}+(\mathbf{u} \cdot \nabla) \mathbf{d} .
$$

Thus, absorbing the gradient term in (3.3) to the pressure, the system (2.13)-(2.14), without the terms related to the anchoring energy, can be rewritten as follows:

$$
\begin{gathered}
\mathbf{d}_{t}+(\mathbf{u} \cdot \nabla) \mathbf{d}=M_{1} K\left(\nabla \cdot\left(\frac{1+\phi}{2}\right)^{2} \nabla \mathbf{d}-\left(\frac{1+\phi}{2}\right)^{2} g(\mathbf{d})\right), \\
\phi_{t}+(\mathbf{u} \cdot \nabla) \phi=M_{2} \lambda(\Delta \phi-f(\phi))-M_{2} K\left(\frac{1+\phi}{2}\right)\left(\frac{|\nabla \mathbf{d}|^{2}}{2}+G(\mathbf{d})\right), \\
\mathbf{u}_{t}+(\mathbf{u} \cdot \nabla) \mathbf{u}-\nu \Delta \mathbf{u}+\nabla p+\frac{\dot{\mathbf{d}}}{M_{1}} \nabla \mathbf{d}+\frac{\dot{\phi}}{M_{2}} \nabla \phi=0, \\
\nabla \cdot \mathbf{u}=0 .
\end{gathered}
$$


We now show that the above system admits an energy law. Taking the inner products of (3.5) with $\frac{\mathrm{d}_{t}}{M_{1}}$, of (3.6) with $\frac{\phi_{t}}{M_{2}}$, and of (3.7) with $\mathbf{u}$, we derive

$$
\begin{aligned}
\frac{\|\dot{\mathbf{d}}\|^{2}}{M_{1}}-\left(\frac{\dot{\mathbf{d}}}{M_{1}},(\mathbf{u} \cdot \nabla) \mathbf{d}\right)= & K\left(\nabla \cdot\left(\frac{1+\phi}{2}\right)^{2} \nabla \mathbf{d}-\left(\frac{1+\phi}{2}\right)^{2} g(\mathbf{d}), \mathbf{d}_{t}\right), \\
\frac{\|\dot{\phi}\|^{2}}{M_{2}}-\left(\frac{\dot{\phi}}{M_{2}},(\mathbf{u} \cdot \nabla) \phi\right)= & -\lambda \partial_{t}\left(\frac{\|\nabla \phi\|^{2}}{2}+(F(\phi), 1)\right) \\
& -K\left(\left(\frac{1+\phi}{2}\right)\left(\frac{|\nabla \mathbf{d}|^{2}}{2}+G(\mathbf{d})\right), \phi_{t}\right)
\end{aligned}
$$

and

$$
\frac{1}{2} \partial_{t}\|\mathbf{u}\|^{2}+\nu\|\nabla \mathbf{u}\|^{2}+\left(\frac{\dot{\mathbf{d}}}{M_{1}} \nabla \mathbf{d}, \mathbf{u}\right)+\left(\frac{\dot{\phi}}{M_{2}} \nabla \phi, \mathbf{u}\right)=0 .
$$

Summing up the above equalities and using the identity

$$
\begin{aligned}
\partial_{t}\left(\left(\frac{1+\phi}{2}\right)^{2}\left(\frac{|\nabla \mathbf{d}|^{2}}{2}+G(\mathbf{d})\right), 1\right)= & \left(\left(\frac{1+\phi}{2}\right)^{2} \nabla \mathbf{d}, \nabla \mathbf{d}_{t}\right)+\left(\left(\frac{1+\phi}{2}\right)^{2} g(\mathbf{d}), \mathbf{d}_{t}\right) \\
& +\left(\left(\frac{1+\phi}{2}\right)\left(\frac{|\nabla \mathbf{d}|^{2}}{2}+G(\mathbf{d})\right), \phi_{t}\right)
\end{aligned}
$$

we arrive at the following result.

Lemma 3.1. Solutions of (3.5)-(3.8) satisfy the following energy law:

$$
\begin{aligned}
\partial_{t} \mathscr{E}_{t o t}= & \partial_{t} \int_{\Omega}\left(\frac{1}{2}|\mathbf{u}|^{2}+\lambda\left(\frac{|\nabla \phi|^{2}}{2}+(F(\phi), 1)\right)\right. \\
& \left.+K\left(\left(\frac{1+\phi}{2}\right)^{2}\left(\frac{|\nabla \mathbf{d}|^{2}}{2}+G(\mathbf{d})\right), 1\right)\right) d x \\
= & -\int_{\Omega}\left(\nu|\nabla \mathbf{u}|^{2}+\frac{|\dot{\mathbf{d}}|^{2}}{M_{1}}+\frac{|\dot{\phi}|^{2}}{M_{2}}\right) d x .
\end{aligned}
$$

We emphasize that the above derivation is suitable in a finite dimensional approximation since test function $\phi_{t}, \mathbf{d}_{t}$ is in the same subspaces as $\phi$ and $\mathbf{d}$. Hence, it allows us to design numerical schemes which satisfy a discrete energy law.

If the Cahn-Hilliard phase equation is used instead of the Allen-Cahn phase equation, we introduce an intermediate variable $\mu$ to split the fourth-order CahnHilliard phase equation into a system of two second-order equations [41, 42]. More precisely, the system with the Cahn-Hilliard phase equation reads as follows:

$$
\begin{aligned}
\mathbf{d}_{t}+(\mathbf{u} \cdot \nabla) \mathbf{d}= & M_{1} K\left(\nabla \cdot\left(\frac{1+\phi}{2}\right)^{2} \nabla \mathbf{d}-\left(\frac{1+\phi}{2}\right)^{2} g(\mathbf{d})\right), \\
& \phi_{t}+(\mathbf{u} \cdot \nabla) \phi=-\Delta \mu,
\end{aligned}
$$




$$
\begin{gathered}
\mu=M_{2} \lambda(\Delta \phi-f(\phi))-M_{2} K\left(\frac{1+\phi}{2}\right)\left(\frac{|\nabla \mathbf{d}|^{2}}{2}+G(\mathbf{d})\right), \\
\mathbf{u}_{t}+(\mathbf{u} \cdot \nabla) \mathbf{u}-\nu \Delta \mathbf{u}+\nabla p+\frac{\dot{\mathbf{d}}}{M_{1}} \nabla \mathbf{d}+\frac{\mu}{M_{2}} \nabla \phi=0, \\
\nabla \cdot \mathbf{u}=0 .
\end{gathered}
$$

Similar to the proof of (3.13), we can establish an energy law for (3.14)-(3.18). Indeed, taking the inner product of (3.14) with $\mathbf{d}_{t} / M_{1}$, of (3.15) with $\mu / M_{2}$, of (3.16) with $\phi_{t}$, and of (3.17) with $\mathbf{u}$, and adding all the results together, we can prove the following.

Lemma 3.2. Solutions of (3.14)-(3.18) satisfy the following energy law:

$$
\begin{aligned}
\partial_{t} \mathscr{E}_{t o t}= & \partial_{t} \int_{\Omega}\left(\frac{1}{2}|\mathbf{u}|^{2}+\lambda\left(\frac{|\nabla \phi|^{2}}{2}+(F(\phi), 1)\right)\right. \\
& \left.+K\left(\left(\frac{1+\phi}{2}\right)^{2}\left(\frac{|\nabla \mathbf{d}|^{2}}{2}+G(\mathbf{d})\right), 1\right)\right) d x \\
= & -\int_{\Omega}\left(\nu|\nabla \mathbf{u}|^{2}+\frac{|\dot{\mathbf{d}}|^{2}}{M_{1}}+\frac{|\nabla \mu|^{2}}{M_{2}}\right) d x .
\end{aligned}
$$

3.2. Energy stable numerical schemes for the Allen-Cahn phase-field model. We first construct an energy stable scheme based on a stabilization approach [42]. To this end, we shall assume that $G(\mathbf{d})$ and $F(\phi)$ satisfy the following conditions: there exists constant $L_{1}$ and $L_{2}$ such that

$$
\max _{|\mathbf{d}| \in \mathbb{R}}|H(\mathbf{d})| \leq L_{1}, \quad \max _{|\phi| \in \mathbb{R}}\left|F^{\prime \prime}(\phi)\right| \leq L_{2},
$$

where $H(\mathbf{d})$ is the Hessian matrix of $G(\mathbf{d})$.

One immediately notes that this condition is not satisfied by the usual GinzburgLandau double-well potential $F(\phi)=\frac{1}{4 \varepsilon^{2}}\left(\phi^{2}-1\right)^{2}$ and $G(\mathbf{d})=\frac{1}{4 \eta^{2}}\left(|\mathbf{d}|^{2}-1\right)^{2}$. However, since it is well known that the Allen-Cahn equation satisfies the maximum principle (for the Cahn-Hilliard equation, a similar result is established in [6]), we can truncate $F(\phi)$ to quadratic growth outside an interval $[-M, M]$ without affecting the solution if the maximum norm of the initial condition $\phi_{0}$ is bounded by $M$. Therefore, it has been a common practice (cf. $[21,8,42]$ ) to consider the Allen-Cahn and Cahn-Hilliard equations with a truncated double-well potential $\tilde{F}(\phi)$. Similarly, we can truncate $G(\mathbf{d})$ to satisfy (3.20).

It is then obvious that there exists $L$ such that (3.20) is satisfied with $F$ replaced by $\tilde{F}$. The same modification is also applied for $g$ in the liquid crystal equation.

Our numerical scheme reads as follows.

Given the initial conditions $\mathbf{d}^{0}, \phi^{0}, \mathbf{u}^{0}$, and $p^{0}=0$, having computed $\mathbf{d}^{n}, \phi^{n}, \mathbf{u}^{n}$, and $p^{n}$ for $n>0$, we compute $\left(\mathbf{d}^{n+1}, \phi^{n+1}, \tilde{\mathbf{u}}^{n+1}, \mathbf{u}^{n+1}, p^{n+1}\right)$ by the following steps.

Step 1.

$$
\left\{\begin{array}{l}
C_{1}^{n}\left(\mathbf{d}^{n+1}-\mathbf{d}^{n}\right)+\frac{1}{M_{1}} \dot{\mathbf{d}}^{n+1}=K \nabla \cdot\left(\frac{1+\phi^{n}}{2}\right)^{2} \nabla \mathbf{d}^{n+1}-K\left(\frac{1+\phi^{n}}{2}\right)^{2} g\left(\mathbf{d}^{n}\right), \\
\left.\frac{\partial \mathbf{d}^{n+1}}{\partial n}\right|_{\partial \Omega}=0,
\end{array}\right.
$$


with

$$
\dot{\mathbf{d}}^{n+1}=\frac{\mathbf{d}^{n+1}-\mathbf{d}^{n}}{\delta t}+\left(\mathbf{u}_{\star}^{n} \cdot \nabla\right) \mathbf{d}^{n}, \quad \mathbf{u}_{\star}^{n}=\mathbf{u}^{n}-\delta t \frac{\dot{\mathbf{d}}^{n+1}}{M_{1}} \nabla \mathbf{d}^{n} .
$$

Step 2.

$$
\left\{\begin{array}{l}
C_{2}^{n}\left(\phi^{n+1}-\phi^{n}\right)+\frac{1}{M_{2}} \dot{\phi}^{n+1}=\lambda\left(\Delta \phi^{n+1}-f\left(\phi^{n}\right)\right)-K\left(\frac{1+\phi^{n+1}}{2}\right) W\left(\mathbf{d}^{n+1}\right), \\
\left.\frac{\partial \phi^{n+1}}{\partial n}\right|_{\partial \Omega}=0
\end{array}\right.
$$

with

$$
\dot{\phi}^{n+1}=\frac{\phi^{n+1}-\phi^{n}}{\delta t}+\left(\mathbf{u}_{\star \star}^{n} \cdot \nabla\right) \phi^{n}, \mathbf{u}_{\star \star}^{n}=\mathbf{u}_{\star}^{n}-\delta t \frac{\dot{\phi}^{n+1}}{M_{2}} \nabla \phi^{n} .
$$

Step 3.

$$
\left\{\begin{array}{l}
\frac{\tilde{\mathbf{u}}^{n+1}-\mathbf{u}^{n}}{\delta t}+\left(\mathbf{u}^{n} \cdot \nabla\right) \tilde{\mathbf{u}}^{n+1}-\nu \Delta \tilde{\mathbf{u}}^{n+1}+\nabla p^{n}+\frac{\dot{\mathbf{d}}^{n+1}}{M_{1}} \nabla \mathbf{d}^{n}+\frac{\dot{\phi}^{n+1}}{M_{2}} \nabla \phi^{n}=0, \\
\left.\tilde{\mathbf{u}}^{n+1}\right|_{\partial \Omega}=0
\end{array}\right.
$$

with

$$
W\left(\mathbf{d}^{n+1}\right)=\left(\frac{\left|\nabla \mathbf{d}^{n+1}\right|^{2}}{2}+G\left(\mathbf{d}^{n+1}\right)\right) .
$$

Step 4.

$$
\left\{\begin{array}{l}
\frac{\mathbf{u}^{n+1}-\tilde{\mathbf{u}}^{n+1}}{\delta t}+\nabla\left(p^{n+1}-p^{n}\right)=0 \\
\nabla \cdot \mathbf{u}^{n+1}=0,\left.n \cdot \mathbf{u}^{n+1}\right|_{\partial \Omega}=0
\end{array}\right.
$$

In the above, $C_{1}^{n}$ and $C_{2}^{n}$ are two stabilizing parameters to be determined.

The above scheme is constructed by combining several effective approaches in approximation of Allen-Cahn equation [42], Navier-Stokes equations [16], and phasefield models $[43,5,37]$.

Several remarks are in order:

- A pressure-correction scheme [16] is used to decouple the computation of the pressure from that of the velocity.

- We recall that $f(\phi)=\frac{1}{\varepsilon^{2}} \phi\left(\phi^{2}-1\right)$, so the explicit treatment of this term usually leads to a severe restriction on the time step $\delta t$ when $\varepsilon \ll 1$. Thus we introduce in (3.23) a stabilizing term to improve stability while preserving simplicity. It allows us to treat the nonlinear term explicitly without suffering from any time step constraint $[43,42,41]$. Note that this stabilizing term introduces an extra consistent error of order $O(\delta t)$ in a small region near the interface, but this error is of the same order as the error introduced by treating $f(\phi)$ explicitly, so the overall truncation error is 
essentially of the same order with or without the stabilizing term. A similar approach is applied to the director equation for the treatment of $g(\mathbf{d})$.

- Inspired by [5, 37], which deal with a phase-field model of three-phase Newtonian fluids, we introduce new, explicit, convective velocities $\mathbf{u}_{\star}^{n}$ and $\mathbf{u}_{\star \star}^{n}$ in the phase equation and director equation. $\mathbf{u}_{\star}^{n}$ and $\mathbf{u}_{\star \star}^{n}$ can be computed directly from (3.22) and (3.24), i.e.,

$$
\mathbf{u}_{\star}^{n}=\frac{\mathbf{u}^{n}-\frac{\mathbf{d}^{n+1}-\mathbf{d}^{n}}{M_{1}} \nabla \mathbf{d}^{n}}{1+\delta t \frac{\left|\nabla \mathbf{d}^{n}\right|^{2}}{M_{1}}}, \quad \mathbf{u}_{\star \star}^{n}=\frac{\mathbf{u}_{\star}^{n}-\frac{\phi^{n+1}-\phi^{n}}{M_{2}} \nabla \phi^{n}}{1+\delta t \frac{\left|\nabla \phi^{n}\right|^{2}}{M_{2}}} .
$$

- The scheme (3.21)-(3.27) is a totally decoupled, linear scheme. Indeed, (3.21), (3.23), and (3.25) are respectively (decoupled) linear elliptic equations for $\mathbf{d}^{n+1}, \phi^{n+1}$, and $\tilde{\mathbf{u}}^{n+1}$, and (3.27) can be reformulated as a Poisson equation for $p^{n+1}-p^{n}$. Therefore, at each time step, one only needs to solve a sequence of decoupled elliptic equations which can be solved very efficiently.

- As we shall show below, the above scheme is unconditionally energy stable. To the best of the authors' knowledge, this is the first such scheme for a model of complex fluids.

THEOREM 3.3. Under the condition (3.20), $C_{1}^{n} \geq K L_{1}\left\|\left(\frac{1+\phi^{n}}{2}\right)^{2}\right\|_{\infty}$, and $C_{2}^{n}=$ $C_{2} \geq \lambda L_{2}$, the scheme (3.21)-(3.27) admits a unique solution satisfying the following discrete energy dissipation law:

$$
\begin{aligned}
\frac{1}{2}\left\|\mathbf{u}^{n+1}\right\|^{2}+\mathbb{E}_{\text {mix }}^{n+1}+\mathbb{E}_{\text {bulk }}^{n+1}+\frac{\delta t^{2}}{2}\left\|\nabla p^{n+1}\right\|^{2} \\
\quad+\left\{\nu \delta t\left\|\nabla \tilde{\mathbf{u}}^{n+1}\right\|^{2}+\delta t\left(\frac{\left|\dot{\phi}^{n+1}\right|^{2}}{M_{2}}+\frac{\left|\dot{\mathbf{d}}^{n+1}\right|^{2}}{M_{1}}\right)\right\} \\
\leq \frac{1}{2}\left\|\mathbf{u}^{n}\right\|^{2}+\mathbb{E}_{m i x}^{n}+\mathbb{E}_{b u l k}^{n}+\frac{\delta t^{2}}{2}\left\|\nabla p^{n}\right\|^{2},
\end{aligned}
$$

where

$$
\begin{aligned}
& \mathbb{E}_{\text {mix }}^{n}=\lambda\left(\frac{1}{2}\left\|\nabla \phi^{n}\right\|^{2}+\left(F\left(\phi^{n}\right), 1\right)\right), \\
& \left.\mathbb{E}_{\text {bulk }}^{n}=K\left(\left(\frac{1+\phi^{n}}{2}\right)^{2}\left(\frac{\left|\nabla \mathbf{d}^{n}\right|^{2}}{2}+G\left(\mathbf{d}^{n}\right)\right), 1\right)\right) .
\end{aligned}
$$

Proof. From the definition of $\mathbf{u}_{\star}^{n}$ and $\mathbf{u}_{\star \star}^{n}$ in (3.22) and (3.24), we can rewrite the momentum equation (3.25) as

$$
\frac{\tilde{\mathbf{u}}^{n+1}-\mathbf{u}_{\star \star}^{n}}{\delta t}+\left(\mathbf{u}^{n} \cdot \nabla\right) \tilde{\mathbf{u}}^{n+1}-\nu \Delta \tilde{\mathbf{u}}^{n+1}+\nabla p^{n}=0 .
$$

By taking the inner product of (3.30) with $2 \delta t \tilde{\mathbf{u}}^{n+1}$, and using the identity

$$
(a-b, 2 a)=|a|^{2}-|b|^{2}+|a-b|^{2},
$$

we obtain

$$
\left\|\tilde{\mathbf{u}}^{n+1}\right\|^{2}-\left\|\mathbf{u}_{\star \star}^{n}\right\|^{2}+\left\|\tilde{\mathbf{u}}^{n+1}-\mathbf{u}_{\star \star}^{n}\right\|^{2}+2 \nu \delta t\left\|\nabla \tilde{\mathbf{u}}^{n+1}\right\|^{2}+2 \delta t\left(\nabla p^{n}, \tilde{\mathbf{u}}^{n+1}\right)=0 .
$$


To deal with the pressure term, we take the inner product of (3.27) with $2 \delta t^{2} \nabla p^{n}$ to derive

$$
\delta t^{2}\left(\left\|\nabla p^{n+1}\right\|^{2}-\left\|\nabla p^{n}\right\|^{2}-\left\|\nabla p^{n+1}-\nabla p^{n}\right\|^{2}\right)=2 \delta t\left(\tilde{\mathbf{u}}^{n+1}, \nabla p^{n}\right) .
$$

By taking the inner product of (3.27) with $\mathbf{u}^{n+1}$, we obtain

$$
\left\|\mathbf{u}^{n+1}\right\|^{2}+\left\|\mathbf{u}^{n+1}-\tilde{\mathbf{u}}^{n+1}\right\|^{2}=\left\|\tilde{\mathbf{u}}^{n+1}\right\|^{2} .
$$

We also derive from (3.27) directly that

$$
\delta t^{2}\left\|\nabla p^{n+1}-\nabla p^{n}\right\|^{2}=\left\|\tilde{\mathbf{u}}^{n+1}-\mathbf{u}^{n+1}\right\|^{2} .
$$

Combining all identities above, we obtain

$\left\|\mathbf{u}^{n+1}\right\|^{2}-\left\|\mathbf{u}_{\star \star}^{n}\right\|^{2}+\left\|\tilde{\mathbf{u}}^{n+1}-\mathbf{u}_{\star \star}^{n}\right\|^{2}+\delta t^{2}\left(\left\|\nabla p^{n+1}\right\|^{2}-\left\|\nabla p^{n}\right\|^{2}\right)+2 \nu \delta t\left\|\nabla \tilde{\mathbf{u}}^{n+1}\right\|^{2}=0$.

Next, we derive from (3.22) and (3.24) that

$$
\begin{aligned}
\frac{\mathbf{u}_{\star}^{n}-\mathbf{u}^{n}}{\delta t} & =-\frac{\dot{\mathbf{d}}^{n+1}}{M_{1}} \nabla \mathbf{d}^{n}, \\
\frac{\mathbf{u}_{\star \star}^{n}-\mathbf{u}_{\star}^{n}}{\delta t} & =-\frac{\dot{\phi}^{n+1}}{M_{2}} \nabla \phi^{n} .
\end{aligned}
$$

By taking the inner product of (3.37) with $2 \delta t \mathbf{u}_{\star}^{n}$, of (3.38) with $2 \delta t \mathbf{u}_{\star \star}^{n}$, we obtain

$$
\begin{aligned}
\left\|\mathbf{u}_{\star}^{n}\right\|^{2}-\left\|\mathbf{u}^{n}\right\|^{2}+\left\|\mathbf{u}_{\star}^{n}-\mathbf{u}_{n}\right\|^{2} & =-2 \delta t\left(\frac{\dot{\mathbf{d}}^{n+1}}{M_{1}} \nabla \mathbf{d}^{n}, \mathbf{u}_{\star}^{n}\right), \\
\left\|\mathbf{u}_{\star \star}^{n}\right\|^{2}-\left\|\mathbf{u}_{\star}^{n}\right\|^{2}+\left\|\mathbf{u}_{\star \star}^{n}-\mathbf{u}_{\star}^{n}\right\|^{2} & =-2 \delta t\left(\frac{\dot{\phi}^{n+1}}{M_{2}} \nabla \phi^{n}, \mathbf{u}_{\star \star}^{n}\right) .
\end{aligned}
$$

Then, by taking the inner product of (3.21) with $2\left(\mathbf{d}^{n+1}-\mathbf{d}^{n}\right)$, we obtain

$$
\begin{aligned}
& 2 C_{1}^{n}\left\|\mathbf{d}^{n+1}-\mathbf{d}^{n}\right\|^{2}+2 \delta t \frac{\left\|\dot{\mathbf{d}}^{n+1}\right\|^{2}}{M_{1}}-2 \delta t\left(\frac{\dot{\mathbf{d}}^{n+1}}{M_{1}},\left(\mathbf{u}_{\star}^{n} \cdot \nabla\right) \mathbf{d}^{n}\right) \\
& +2 K\left(\left(\frac{1+\phi^{n}}{2}\right)^{2} g\left(\mathbf{d}^{n}\right), \mathbf{d}^{n+1}-\mathbf{d}^{n}\right) \\
& +2 K\left(\left(\frac{1+\phi^{n}}{2}\right)^{2} \nabla \mathbf{d}^{n+1}, \nabla \mathbf{d}^{n+1}-\nabla \mathbf{d}^{n}\right)=0 .
\end{aligned}
$$

By taking the inner product of (3.23) with $2\left(\phi^{n+1}-\phi^{n}\right)$, we derive

$$
\begin{aligned}
2 C_{2}^{n}\left\|\phi^{n+1}-\phi^{n}\right\|^{2}+2 \delta t \frac{\left\|\dot{\phi}^{n+1}\right\|^{2}}{M_{2}}-2 \delta t\left(\frac{\dot{\phi}^{n+1}}{M_{2}},\left(\mathbf{u}_{\star \star}^{n} \cdot \nabla\right) \phi^{n}\right) \\
\quad+2 \lambda\left(\frac{\left\|\nabla \phi^{n+1}\right\|^{2}}{2}-\frac{\left\|\nabla \phi^{n}\right\|^{2}}{2}+\frac{\left\|\nabla \phi^{n+1}-\nabla \phi^{n}\right\|^{2}}{2}\right) \\
+2 \lambda\left(f\left(\phi^{n}\right), \phi^{n+1}-\phi^{n}\right)+2 K\left(\left(\frac{1+\phi^{n+1}}{2}\right) W\left(\mathbf{d}^{n+1}\right), \phi^{n+1}-\phi^{n}\right)=0 .
\end{aligned}
$$


Combining (3.36), (3.39), (3.40), (3.41), and (3.42), we arrive at

$$
\begin{aligned}
& \left\|\mathbf{u}^{n+1}\right\|^{2}-\left\|\mathbf{u}^{n}\right\|^{2}+\left\|\tilde{\mathbf{u}}^{n+1}-\mathbf{u}_{\star \star}^{n}\right\|^{2}+\left\|\mathbf{u}_{\star \star}^{n}-\mathbf{u}_{\star}^{n}\right\|^{2} \\
& + \\
& +\left\|\mathbf{u}_{\star}^{n}-\mathbf{u}^{n}\right\|^{2}+\delta t^{2}\left(\left\|\nabla p^{n+1}\right\|^{2}-\left\|\nabla p^{n}\right\|^{2}\right) \\
& \quad+2 \nu \delta t\left\|\nabla \tilde{\mathbf{u}}^{n+1}\right\|^{2}+2 \delta t \frac{\left\|\dot{\mathbf{d}}^{n+1}\right\|^{2}}{M_{1}}+2 \delta t \frac{\left\|\dot{\phi}^{n+1}\right\|^{2}}{M_{2}} \\
& \quad+2 \lambda\left(\frac{\left\|\nabla \phi^{n+1}\right\|^{2}}{2}-\frac{\left\|\nabla \phi^{n}\right\|^{2}}{2}+\frac{\left\|\nabla \phi^{n+1}-\nabla \phi^{n}\right\|^{2}}{2}\right) \\
& \quad+2 C_{1}^{n}\left\|\mathbf{d}^{n+1}-\mathbf{d}^{n}\right\|^{2}+2 C_{2}^{n}\left\|\phi^{n+1}-\phi^{n}\right\|^{2} \\
& \quad+2 \lambda\left(f\left(\phi^{n}\right), \phi^{n+1}-\phi^{n}\right) \quad(\text { Term } A) \\
& +2 K\left(\left(\frac{1+\phi^{n+1}}{2}\right) W\left(\mathbf{d}^{n+1}\right), \phi^{n+1}-\phi^{n}\right) \quad(\text { Term } B) \\
& +2 K\left(\left(\frac{1+\phi^{n}}{2}\right)^{2} \nabla \mathbf{d}^{n+1}, \nabla \mathbf{d}^{n+1}-\nabla \mathbf{d}^{n}\right) \quad(\text { Term } C) \\
& +2 K\left(\left(\frac{1+\phi^{n}}{2}\right)^{2} g\left(\mathbf{d}^{n}\right), \mathbf{d}^{n+1}-\mathbf{d}^{n}\right) \quad(\text { Term } D)=0 .
\end{aligned}
$$

We deal with Terms $A, B, C, D$ as follows.

For Term $A$, we apply the Taylor expansions to obtain

$$
A=2 \lambda\left(F\left(\phi^{n+1}\right)-F\left(\phi^{n}\right), 1\right)-2 \lambda f^{\prime}(\xi)\left\|\phi^{n+1}-\phi^{n}\right\|^{2} .
$$

For Term $B$, we have

$$
\begin{aligned}
B & =4 K\left(W\left(\mathbf{d}^{n+1}\right)\left(\frac{1+\phi^{n+1}}{2}\right),\left(\frac{1+\phi^{n+1}}{2}\right)-\left(\frac{1+\phi^{n}}{2}\right)\right) \\
& =2 K\left(W\left(\mathbf{d}^{n+1}\right),\left(\frac{1+\phi^{n+1}}{2}\right)^{2}-\left(\frac{1+\phi^{n}}{2}\right)^{2}\right)+2 K\left(W\left(\mathbf{d}^{n+1}\right),\left(\frac{\phi^{n+1}-\phi^{n}}{2}\right)^{2}\right) .
\end{aligned}
$$

For Term $C$, we have

$$
\begin{aligned}
C= & 2 K\left(\left(\frac{1+\phi^{n}}{2}\right)^{2}, \frac{\left(\nabla \mathbf{d}^{n+1}\right)^{2}}{2}-\frac{\left(\nabla \mathbf{d}^{n}\right)^{2}}{2}\right) \\
& +2 K\left(\left(\frac{1+\phi^{n+1}}{2}\right)^{2}, \frac{\left(\nabla \mathbf{d}^{n+1}-\nabla \mathbf{d}^{n}\right)^{2}}{2}\right) .
\end{aligned}
$$

For Term $D$, we apply the Taylor expansion to derive

$$
D=2 K\left(\left(\frac{1+\phi^{n}}{2}\right)^{2}, G\left(\mathbf{d}^{n+1}\right)-G\left(\mathbf{d}^{n}\right)\right)-2 K\left(\left(\frac{1+\phi^{n}}{2}\right)^{2}, H(\zeta)\left(\mathbf{d}^{n+1}-\mathbf{d}^{n}\right)^{2}\right) .
$$


Finally, combining (3.43), (3.44)-(3.47), and dropping some positive terms, we obtain

$$
\begin{aligned}
& \left\|\mathbf{u}^{n+1}\right\|^{2}-\left\|\mathbf{u}^{n}\right\|^{2}+\delta t^{2}\left(\left\|\nabla p^{n+1}\right\|^{2}-\left\|\nabla p^{n}\right\|^{2}\right)+2 \nu \delta t\left\|\nabla \tilde{\mathbf{u}}^{n+1}\right\|^{2} \\
& +2 \delta\left(\frac{\left|\dot{\mathbf{d}}^{n+1}\right|^{2}}{M_{1}}+\frac{\left|\dot{\phi}^{n+1}\right|^{2}}{M_{2}}\right)+2 \lambda\left(\frac{\left\|\nabla \phi^{n+1}\right\|^{2}}{2}-\frac{\left\|\nabla \phi^{n}\right\|^{2}}{2}\right) \\
& +2 \lambda\left(F\left(\phi^{n+1}\right)-F\left(\phi^{n}\right), 1\right) \\
& +2 K\left(W\left(\mathbf{d}^{n+1}\right),\left(\frac{1+\phi^{n+1}}{2}\right)^{2}\right)-\left(W\left(\mathbf{d}^{n}\right),\left(\frac{1+\phi^{n}}{2}\right)^{2}\right) \\
& \quad+\left(2 C_{1}^{n}-2 K L_{1}\left\|\left(\frac{1+\phi^{n}}{2}\right)^{2}\right\|_{\infty}\right)\left\|\mathbf{d}^{n+1}-\mathbf{d}^{n}\right\|^{2} \\
& +\left(2 C_{2}^{n}-2 \lambda L_{2}\right)\left\|\phi^{n+1}-\phi^{n}\right\|^{2} \leq 0,
\end{aligned}
$$

which implies the desired result.

Next, we construct a similar scheme based the convex-splitting method [11, 14].

Let us denote $F_{c}(x)=\frac{x^{4}+1}{\varepsilon^{2}}, F_{e}(x)=\frac{2 x^{2}}{\varepsilon^{2}}$ and $G_{c}(x)=\frac{x^{4}+1}{\eta^{2}}, G_{e}(x)=\frac{2 x^{2}}{\eta^{2}}$. Then $F(x)$ and $G(x)$ can be split into a difference of two convex functions, i.e.,

$$
F(x)=F_{c}(x)-F_{e}(x), \quad G(x)=G_{c}(x)-G_{e}(x),
$$

with $F_{c}^{\prime \prime}(x), F_{e}^{\prime \prime}(x) \geq 0$. We define

$$
f_{c}(x)=F_{c}^{\prime}(x), \quad f_{e}(x)=F_{e}^{\prime}(x), \quad g_{c}(x)=\nabla G_{c}(x), \quad g_{e}(x)=\nabla G_{e}(x) .
$$

The scheme with convex-splitting is obtained by replacing (3.21) and (3.23) with

$$
\left\{\begin{array}{l}
\frac{1}{M_{1}} \dot{\mathbf{d}}^{n+1}=K \nabla \cdot\left(\frac{1+\phi^{n}}{2}\right)^{2} \nabla \mathbf{d}^{n+1}-K\left(\frac{1+\phi^{n}}{2}\right)^{2}\left(g_{c}\left(\mathbf{d}^{n+1}\right)-g_{e}\left(\mathbf{d}^{n}\right)\right), \\
\left.\frac{\partial \mathbf{d}^{n+1}}{\partial n}\right|_{\partial \Omega}=0
\end{array}\right.
$$

and

$$
\left\{\begin{array}{l}
\frac{1}{M_{2}} \dot{\phi}^{n+1}=\lambda\left(\Delta \phi^{n+1}-f_{c}\left(\phi^{n+1}\right)+f_{e}\left(\phi^{n}\right)\right)-K\left(\frac{1+\phi^{n+1}}{2}\right) W\left(\mathbf{d}^{n+1}\right) \\
\left.\frac{\partial \phi^{n+1}}{\partial n}\right|_{\partial \Omega}=0
\end{array}\right.
$$

where $\dot{\mathbf{d}}^{n+1}$ and $\dot{\phi}^{n+1}$ are defined in (3.22) and (3.24).

We note that the above equations are decoupled but nonlinear. However, it is easy to show that $\mathbf{d}$ and $\phi$ are the unique minimizer of a convex functional (cf., for instance, $[44,33])$.

For this scheme, the following result can be established by following a similar procedure in the proof of Theorem 3.3. Details are left to the interested reader. 
Corollary 3.4. The scheme (3.51)-(3.52)-(3.25)-(3.27) admits a unique solution satisfying the following discrete energy law:

$$
\begin{aligned}
\frac{1}{2}\left\|\mathbf{u}^{n+1}\right\|_{L^{2}}^{2}+\frac{\delta t^{2}}{2}\left\|\nabla p^{n+1}\right\|_{L^{2}}^{2}+\mathbb{E}_{m i x}^{n+1}+\mathbb{E}_{b u l k}^{n+1} & \\
+ & \left\{\nu \delta t\left\|\nabla \tilde{\mathbf{u}}^{n+1}\right\|^{2}+\delta t\left(\frac{\left|\dot{\phi}^{n+1}\right|^{2}}{M_{2}}+\frac{\left|\dot{\mathbf{d}}^{n+1}\right|^{2}}{M_{1}}\right)\right\} \\
\leq & \frac{1}{2}\left\|\mathbf{u}^{n}\right\|_{L^{2}}^{2}+\frac{\delta t^{2}}{2}\left\|\nabla p^{n}\right\|_{L^{2}}^{2}+\mathbb{E}_{m i x}^{n}+\mathbb{E}_{b u l k}^{n},
\end{aligned}
$$

where $\mathbb{E}_{\text {mix }}^{n}$ and $\mathbb{E}_{\text {bulk }}^{n}$ are defined in (3.29).

3.3. Energy stable numerical schemes for the Cahn-Hilliard phase-field model. We now consider the phase-field model with the Cahn-Hilliard phase equation (3.14)-(3.18). Similarly, we can construct numerical schemes enjoying the same properties as those for the Allen-Cahn phase-field model. More precisely, the totally decoupled linear scheme based on the stabilization reads as follows:

Step 1.

$$
\left\{\begin{array}{l}
C_{1}^{n}\left(\mathbf{d}^{n+1}-\mathbf{d}^{n}\right)+\frac{1}{M_{1}} \dot{\mathbf{d}}^{n+1}=K \nabla \cdot\left(\frac{1+\phi^{n}}{2}\right)^{2} \nabla \mathbf{d}^{n+1}-K\left(\frac{1+\phi^{n}}{2}\right)^{2} g\left(\mathbf{d}^{n}\right), \\
\left.\frac{\partial \mathbf{d}^{n+1}}{\partial n}\right|_{\partial \Omega}=0,
\end{array}\right.
$$

with

$$
\dot{\mathbf{d}}^{n+1}=\frac{\mathbf{d}^{n+1}-\mathbf{d}^{n}}{\delta t}+\left(\mathbf{u}_{\star}^{n} \cdot \nabla\right) \mathbf{d}^{n}, \quad \mathbf{u}_{\star}^{n}=\mathbf{u}^{n}-\delta t \frac{\dot{\mathbf{d}}^{n+1}}{M_{1}} \nabla \mathbf{d}^{n} .
$$

Step 2.

$$
\left\{\begin{array}{l}
\dot{\phi}^{n+1}=-\Delta \mu^{n+1} \\
C_{2}^{n}\left(\phi^{n+1}-\phi^{n}\right)+\frac{1}{M_{2}} \mu^{n+1}=\lambda\left(\Delta \phi^{n+1}-f\left(\phi^{n}\right)\right)-K\left(\frac{1+\phi^{n+1}}{2}\right) W\left(\mathbf{d}^{n+1}\right) \\
\left.\frac{\partial \mu^{n+1}}{\partial n}\right|_{\partial \Omega}=0,\left.\frac{\partial \phi^{n+1}}{\partial n}\right|_{\partial \Omega}=0
\end{array}\right.
$$

with

$$
\dot{\phi}^{n+1}=\frac{\phi^{n+1}-\phi^{n}}{\delta t}+\left(\mathbf{u}_{\star \star}^{n} \cdot \nabla\right) \phi^{n}, \mathbf{u}_{\star \star}^{n}=\mathbf{u}_{\star}^{n}-\delta t \frac{\mu^{n+1}}{M_{2}} \nabla \phi^{n} .
$$

Step 3.

$$
\left\{\begin{array}{l}
\frac{\tilde{\mathbf{u}}^{n+1}-\mathbf{u}^{n}}{\delta t}+\left(\mathbf{u}^{n} \cdot \nabla\right) \tilde{\mathbf{u}}^{n+1}-\nu \Delta \tilde{\mathbf{u}}^{n+1}+\nabla p^{n}+\frac{\dot{\mathbf{d}}^{n+1}}{M_{1}} \nabla \mathbf{d}^{n}+\frac{\mu^{n+1}}{M_{2}} \nabla \phi^{n}=0, \\
\left.\tilde{\mathbf{u}}^{n+1}\right|_{\partial \Omega}=0
\end{array}\right.
$$


with

$$
W\left(\mathbf{d}^{n+1}\right)=\left(\frac{\left|\nabla \mathbf{d}^{n+1}\right|^{2}}{2}+G\left(\mathbf{d}^{n+1}\right)\right) .
$$

Step 4.

$$
\left\{\begin{array}{l}
\frac{\mathbf{u}^{n+1}-\tilde{\mathbf{u}}^{n+1}}{\delta t}+\nabla\left(p^{n+1}-p^{n}\right)=0, \\
\nabla \cdot \mathbf{u}^{n+1}=0,\left.n \cdot \mathbf{u}^{n+1}\right|_{\partial \Omega}=0 .
\end{array}\right.
$$

For the above scheme, we have the following result.

THEOREM 3.5. Under the condition (3.20), $C_{1}^{n} \geq K L_{1}\left\|\left(\frac{1+\phi^{n}}{2}\right)^{2}\right\|_{\infty}$, and $C_{2}^{n}=$ $C_{2} \geq \lambda L_{2}$, the scheme (3.53)-(3.59) admits a unique solution satisfying the following discrete energy dissipation law:

$$
\begin{aligned}
\frac{1}{2}\left\|\mathbf{u}^{n+1}\right\|^{2}+\mathbb{E}_{m i x}^{n+1}+\mathbb{E}_{\text {bulk }}^{n+1}+\frac{\delta t^{2}}{2}\left\|\nabla p^{n+1}\right\|^{2} \\
\quad+\left\{\nu \delta t\left\|\nabla \tilde{\mathbf{u}}^{n+1}\right\|^{2}+\delta t\left(\frac{\left|\nabla \mu^{n+1}\right|^{2}}{M_{2}}+\frac{\left|\dot{\mathbf{d}}^{n+1}\right|^{2}}{M_{1}}\right)\right\} \\
\leq \frac{1}{2}\left\|\mathbf{u}^{n}\right\|^{2}+\mathbb{E}_{m i x}^{n}+\mathbb{E}_{b u l k}^{n}+\frac{\delta t^{2}}{2}\left\|\nabla p^{n}\right\|^{2},
\end{aligned}
$$

where $\mathbb{E}_{\text {mix }}^{n}$ and $\mathbb{E}_{\text {bulk }}^{n}$ are defined in (3.29).

Proof. The proof is similar to that of Theorem 3.3, so we only sketch their main differences below.

Instead of (3.42), we take the inner product of the first equation of (3.55) with $-2 \delta t \frac{\mu^{n+1}}{M_{2}}$ and of the second equation with $2\left(\phi^{n+1}-\phi^{n}\right)$, respectively, to obtain

$$
-2 \delta t\left(\frac{\mu^{n+1}}{M_{2}}, \dot{\phi}^{n+1}\right)=-2 \delta t \frac{\left\|\nabla \mu^{n+1}\right\|^{2}}{M_{2}}
$$

and

$$
\begin{aligned}
& 2 C_{2}^{n}\left\|\phi^{n+1}-\phi^{n}\right\|^{2}+2 \delta t\left(\frac{\mu^{n+1}}{M_{2}}, \dot{\phi}^{n+1}\right)-2 \delta t\left(\frac{\mu^{n+1}}{M_{2}},\left(\mathbf{u}_{\star \star}^{n} \cdot \nabla\right) \phi^{n}\right) \\
& \quad+2 \lambda\left(\frac{\left\|\nabla \phi^{n+1}\right\|^{2}}{2}-\frac{\left\|\nabla \phi^{n}\right\|^{2}}{2}+\frac{\left\|\nabla \phi^{n+1}-\nabla \phi^{n}\right\|^{2}}{2}\right) \\
& \quad+2 \lambda\left(f\left(\phi^{n}\right), \phi^{n+1}-\phi^{n}\right)+2 K\left(\left(\frac{1+\phi^{n+1}}{2}\right) W\left(\mathbf{d}^{n+1}\right), \phi^{n+1}-\phi^{n}\right)=0 .
\end{aligned}
$$

Summing up the above two equalities, we arrive at

$$
\begin{aligned}
& 2 C_{2}^{n}\left\|\phi^{n+1}-\phi^{n}\right\|^{2}+2 \delta t \frac{\left\|\nabla \mu^{n+1}\right\|^{2}}{M_{2}}-2 \delta t\left(\frac{\mu^{n+1}}{M_{2}},\left(\mathbf{u}_{\star \star}^{n} \cdot \nabla\right) \phi^{n}\right) \\
& \quad+2 \lambda\left(\frac{\left\|\nabla \phi^{n+1}\right\|^{2}}{2}-\frac{\left\|\nabla \phi^{n}\right\|^{2}}{2}+\frac{\left\|\nabla \phi^{n+1}-\nabla \phi^{n}\right\|^{2}}{2}\right) \\
& \quad+2 \lambda\left(f\left(\phi^{n}\right), \phi^{n+1}-\phi^{n}\right)+2 K\left(\left(\frac{1+\phi^{n+1}}{2}\right) W\left(\mathbf{d}^{n+1}\right), \phi^{n+1}-\phi^{n}\right)=0 .
\end{aligned}
$$


The above relation should replace (3.42) in the proof of Theorem 3.3. The other parts of the proof are essentially the same as the proof of Theorem 3.3.

Instead of the stabilized approach in (3.53) and (3.55), we can also use the convex-splitting approach. More precisely, we can replace (3.53) by (3.51) and replace (3.55) by

$$
\left\{\begin{array}{l}
\dot{\phi}^{n+1}=-\Delta \mu^{n+1},\left.\quad \frac{\partial \mu^{n+1}}{\partial n}\right|_{\partial \Omega}=0 \\
\frac{1}{M_{2}} \mu^{n+1}=\lambda\left(\Delta \phi^{n+1}-f_{c}\left(\phi^{n+1}\right)+f_{e}\left(\phi^{n}\right)\right) \\
-K\left(\frac{1+\phi^{n+1}}{2}\right) W\left(\mathbf{d}^{n+1}\right),\left.\quad \frac{\partial \phi^{n+1}}{\partial n}\right|_{\partial \Omega}=0
\end{array}\right.
$$

where $\dot{\phi}^{n+1}$ is defined in (3.56).

Then, using the same arguments in the proof of Theorem 3.5, we can prove the following.

Corollary 3.6. The scheme (3.51)-(3.63)-(3.57)-(3.59) admits a unique solution satisfying the following discrete energy dissipation law:

$$
\begin{aligned}
\frac{1}{2}\left\|\mathbf{u}^{n+1}\right\|^{2}+\mathbb{E}_{m i x}^{n+1}+\mathbb{E}_{b u l k}^{n+1}+\frac{\delta t^{2}}{2}\left\|\nabla p^{n+1}\right\|^{2} \\
\quad+\left\{\nu \delta t\left\|\nabla \tilde{\mathbf{u}}^{n+1}\right\|^{2}+\delta t\left(\frac{\left|\nabla \mu^{n+1}\right|^{2}}{M_{2}}+\frac{\left|\dot{\mathbf{d}}^{n+1}\right|^{2}}{M_{1}}\right)\right\} \\
\leq \frac{1}{2}\left\|\mathbf{u}^{n}\right\|^{2}+\mathbb{E}_{\text {mix }}^{n}+\mathbb{E}_{b u l k}^{n}+\frac{\delta t^{2}}{2}\left\|\nabla p^{n}\right\|^{2}
\end{aligned}
$$

where $\mathbb{E}_{\text {mix }}^{n}$ and $\mathbb{E}_{\text {bulk }}^{n}$ are defined in (3.29).

4. Numerical simulations. We present in this section some numerical experiments by using the scheme (3.21)-(3.27) to solve the Allen-Cahn type complex fluid model in a three-dimensional cylinder with axisymmetry. A Lagrange multiplier is added in (3.23) to enforce the conservation of volume fraction. For the space discretization, we use the Legendre-Galerkin method [39, 40]. For more details on the Legendre-Galerkin method for solving the vector (for the velocity and director) and scalar (for the pressure and phase function) Poisson type equations in a cylindrical axisymmetric geometry, we refer to [35, 47]. In numerical simulations, after we preassign the interfacial width $\varepsilon$, the grid resolution is decided to ensure that the interfaces are fully resolved, and the time step is set to obtain the desirable accuracy.

Below, we present several numerical experiments. In these simulations, the computational domain is an axisymmetric cylinder of radius of $R$ and height $H: \Omega=$ $\{(r, z): r \in(0, R), z \in(0, H)\}$. In all simulations, the following parameters are used:

$$
\nu=1, M_{1}=0.01, M_{2}=0.1, \lambda=0.001 ; \varepsilon=0.01, \eta=0.03, \delta t=0.0001 .
$$

The initial velocity and pressure are set to zero. A $512 \times 512$ spatial discretization is used.

To illustrate that our numerical scheme indeed obeys the discrete energy law proved in the last section, we have plotted the evolution of the discrete total energy, 

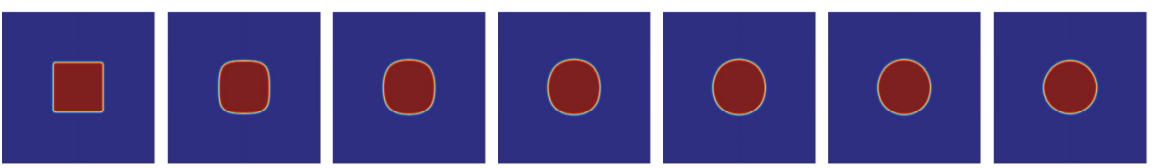

FIG. 1. The dynamics of a cylindrical, viscous filament immersed in viscous fluids. Snapshots are shown at $t=0,0.25,0.5,0.75,1,1.25,3.5$.

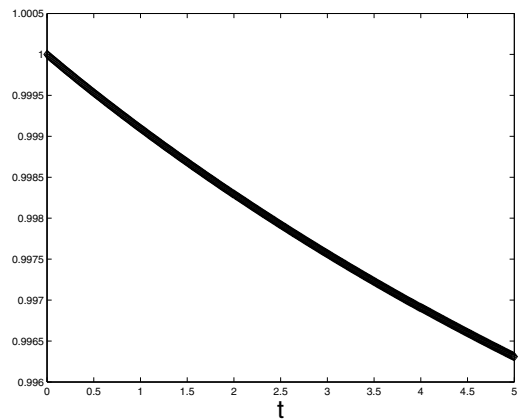

FIG. 2. The energy plot of Example 1.

$\mathbb{E}_{\text {tot }}^{n} / \mathbb{E}_{\text {tot }}^{0}$, where $\mathbb{E}_{\text {tot }}^{n}=\frac{1}{2}\left\|\mathbf{u}^{n}\right\|^{2}+\mathbb{E}_{\text {mix }}^{n}+\mathbb{E}_{\text {bulk }}^{n}+\frac{\delta t^{2}}{2}\left\|\nabla p^{n}\right\|^{2}$, for the first four examples below. One observes from these plots that the discrete total energy indeed decays with time.

Example 1. The dynamics of a cylindrical, viscous filament in a viscous fluid. We first simulate the evolution of a "fat," cylindrical, viscous filament of radius $R_{0}=R / 3$ and height $H_{0}=2 R / 3$ centered around the axis of a cylinder or radius $R=1$ and $H=2$. The rest of the cylinder is filled with an immiscible ambient viscous fluid of the same density and viscosity. This is the viscous limit of our model (3.5)-(3.8) when $K=0$. As expected, this filament quickly deforms into a sphere due to the surface tension (Figure 1). The energy dissipation curve is showed in Figure 2.

Example 2. The dynamics of a cylindrical, liquid crystal filament (with $A=0$ ) in a viscous fluid. In this test, we choose the same initial condition for the phase function $\phi$ as in Example 1. The initial condition for $\mathbf{d}$ is set to be $\mathbf{d}_{0}=0 e_{r}+\left(\frac{1+\phi}{2}\right)^{2} e_{z}$, where $e_{r}, e_{z}$ is the unit vector on the $(r, z)$ direction. The profile of the $\mathbf{d}_{0}$ is shown in Figure $3(\mathrm{a})$. We set $K=1$ in this example. Notice that an undulation appears before the filament finally evolves into a sphere. The undulation is the result of the competition between the surface tension and the elastic energy of liquid crystal (Figure 3(b)). The final profile of the director field $\mathbf{d}$ is shown in Figure 3(c). The energy dissipation curve is shown in Figure 4.

Example 3. The dynamics of a cylindrical, liquid crystal filament with $A \neq 0$ in a viscous fluid. In this example, we examine the effects of the anchoring energy. The initial condition of phase function $\phi$ and $\mathbf{d}$ are the same as in Example 2. The bulk energy constant is still $K=1$ and the anchoring energy constant is set to be $A=3$. Comparing to Example 2, the undulation is now much less pronounced due to the anchoring effects of the liquid crystal which help to stabilize the deformation, and the final shape of the filament is a ellipsoid instead of a sphere due to the anchoring effects (Figure 5(b)). The final profile of the director field is shown in Figure 5(c). The energy dissipation curve is shown in Figure 6. 




(a) The initial profile of the director field $\mathbf{d}_{0}$.
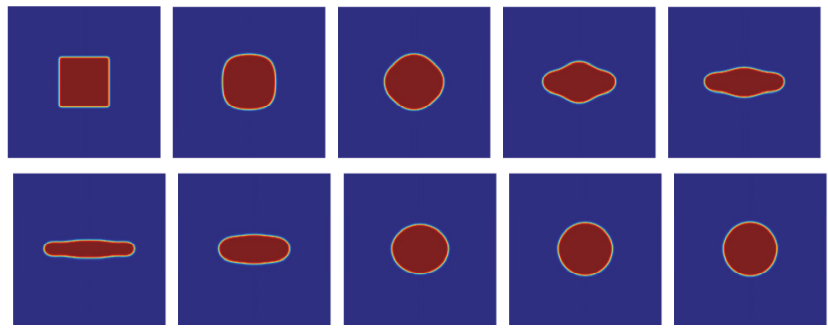

(b)

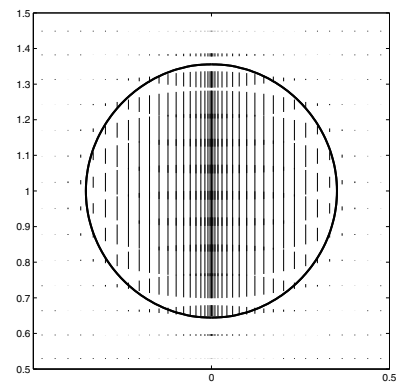

(c) The profile of the director field $\mathbf{d}$ at steady state.

FIG. 3. The dynamics of a viscous cube in viscous fluids. Snapshots are shown at $t=$ $0,0.02,0.04,0.08,0.1,0.2,0.5,1,1.2,1.4$.

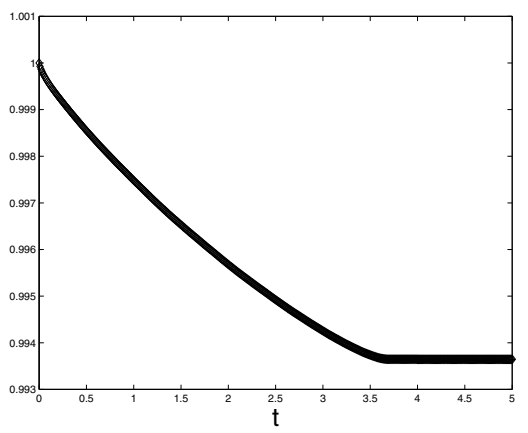

FIG. 4. The energy plot of Example 2. 




(a) The initial profile of the director field $\mathbf{d}_{0}$.
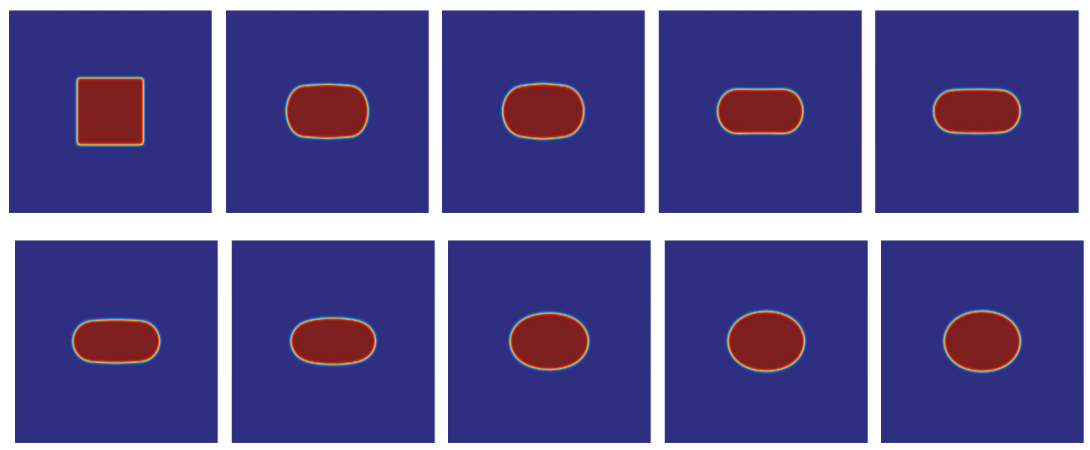

(b) The dynamics of the phase field function $\phi$. Snapshots are shown at $t=$ $0,0.05,0.2,0.3,0.4,0.5,1,2,3,3.5$.

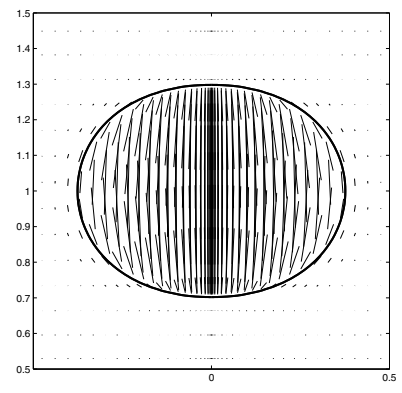

(c) The profile of the director field $\mathbf{d}$ at steady state.

FIG. 5. The dynamics of a liquid crystal cube with $K=1, A=3$.

Example 4. The dynamics of a spherical, viscous drop in a liquid crystal fluid. We start with a viscous spherical drop of radius $R / 3$ that is immersed in a liquid crystal fluid. In this example, $\phi=1$ (ambient fluid) indicates the liquid cystal fluid and $\phi=-1$ (drop) represents the viscous fluid. The initial condition of the director field is still $\mathbf{d}_{0}=0 e_{r}+\left(\frac{1+\phi}{2}\right)^{2} e_{z}$. Figure $7\left(\right.$ a) represents the initial director field $\mathbf{d}_{0}$ that is only nonzero outside the bubble. We set the bulk energy constant $K=1$ and the anchoring energy constant $A=3$. As time evolves, the ambient liquid crystal fluid squeezes the viscous drop into an ellipsoid shape (Figure 7(b)). This is similar and consistent to the experiment reported in [1] and the numerical simulation in [50]. 


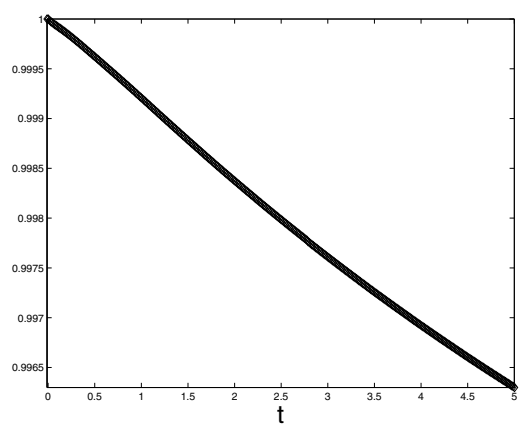

FIG. 6. The energy plot of Example 3.

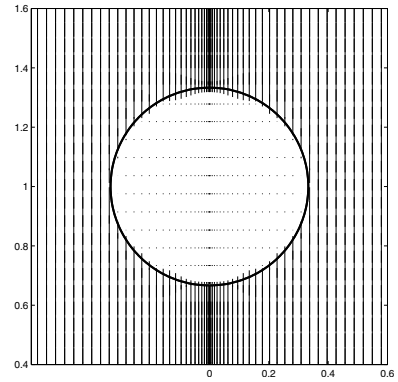

(a) The initial profile of the director field $\mathbf{d}_{0}$.
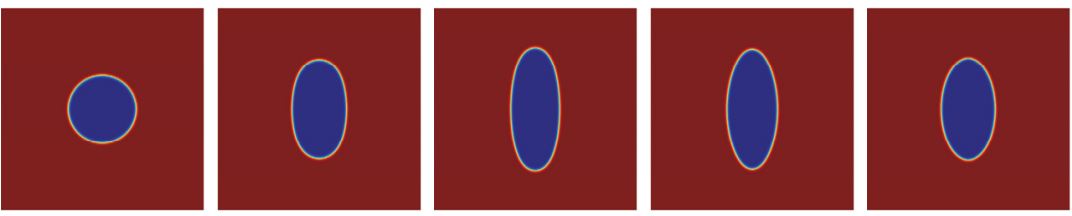

(b) Snapshots at $t=0,0.1,0.25,0.5,1$.

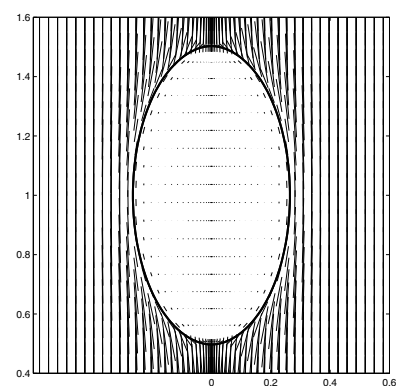

(c) The profile of the director field $\mathbf{d}$ at steady state.

FIG. 7. The dynamics of a viscous drop immersed in liquid crystal fluid with $K=1, A=3$. 


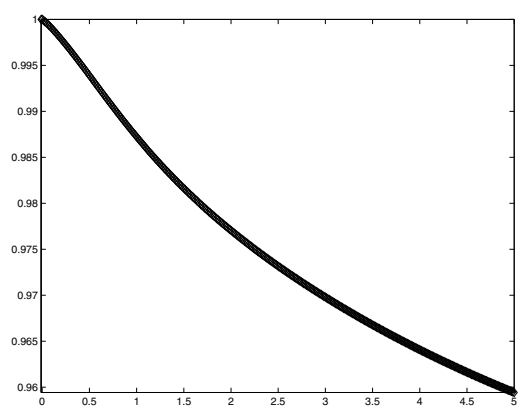

FIG. 8. The energy plot of Example 4.

The final profile of the director field is shown in Figure 7(c). The energy dissipation curve is showed in Figure 8.

Example 5. The dynamics of a falling liquid crystal drop. We start with a liquid crystal drop immersed in a viscous fluid by gravity. We consider the case where the density difference of the liquid crystal drop and ambient fluid is small so that we can use the Boussinesq approximation $[32,47]$ in the momentum equation as follows:

$$
\rho_{0}\left(\mathbf{u}_{t}+(\mathbf{u} \cdot \nabla) \mathbf{u}\right)+\nabla p=\nabla \cdot \sigma+f_{g t y} e_{z},
$$

where $f_{\text {gty }}=-\left((1+\phi)\left(\rho_{1}-\rho_{0}\right)+(1-\phi)\left(\rho_{2}-\rho_{0}\right)\right) g_{0}, g_{0}$ is the gravity acceleration. We set $\rho_{0}=1, \rho_{1}=0.5, \rho_{2}=1.5$, and $g_{0}=10$. The computation domain is $R=1, H=6$. The initial conditions are set to be

$$
\begin{aligned}
& \phi_{0}(r, z)=\tanh \left(\frac{R_{0}^{2}-r^{2}-(z-H-0.6)^{2}}{\varepsilon}\right), \\
& \mathbf{d}_{0}(r, z)=0 e_{r}+\left(\frac{1+\phi_{0}}{2}\right)^{2} e_{z},
\end{aligned}
$$

where $R_{0}=0.96$. We set $K=1, A=0$. Figure 9 represents the deformation dynamics of the falling drop. The elastic bulk energy leads to the formation of a persistent, long thread connecting the liquid crystals at the faucet and the liquid crystal drop that forms at the leading edge of the falling drop. Instead of breaking up, the thin thread forms a smaller satellite drop. In Figure 10, we compare some snapshots of our simulation results together with the experimental results in [45].

Example 6. The dynamics of a larger falling liquid crystal drop. We consider the same senario as in Example 5, except that we slightly enlarged the initial drop.


FIG. 9. The dynamics of the deformation of a liquid crystal drop that is immersed in a viscous fluid at $t=0,4,6,8,9,9.6,10,10.2,10.4,10.6$ with $K=1, A=0$. 

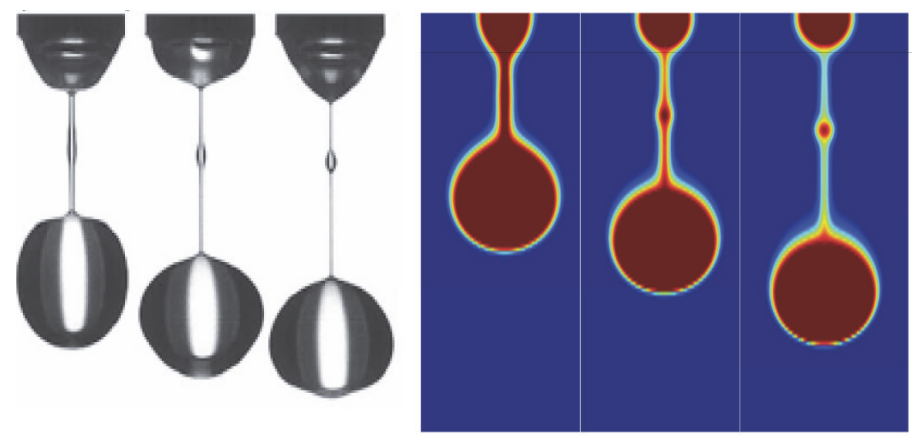

FIG. 10. Qualitative comparison between the experimental benchmark [45] of viscoelastic fluid (left, 0.1 wt. $\% 1 \times 10^{6} \mathrm{~g} / \mathrm{mol}$ PEO in solvent of $36 \%$ glyercol) and our simulations (right) in Figure 9 of $t=10.2,10.4,10.6$.


FIG. 11. The dynamics of the deformation of a liquid crystal drop immersed in a viscous fluid at $t=0,1,2,3,3.4,4,4.4,5$ with $K=1, A=0$.

Let $\phi_{0}(r, z)=\tanh \left(\frac{R_{0}^{2}-r^{2}-(z-H-0.45)^{2}}{\varepsilon}\right)$, and all other parameters are the same as in Example 5. Figure 11 represents the deformation and pinch-off process for this larger falling drop. The dynamics are similar to Example 5 but it forms more satellite drops and a longer thread.

5. Summary. We studied in this paper energetic, Allen-Cahn and Cahn-Hilliard, phase-field models for two-phase, one liquid crystal phase, and one viscous fluid phase complex fluids. We first reformulated the models to a form which is suitable for numerical approximation and derived the associated energy dissipation laws. We then constructed two classes of numerical schemes, one based on a stabilized technique and the other based on a convex-splitting approach. These schemes enjoy the following properties: (i) they lead to completely decoupled elliptic equations to solve at each time step; (ii) they are unconditionally stable and obey a discrete energy law; and (iii) in the case of stabilized approach, all elliptic equations are linear, while in the case of convex-splitting approach, the solutions of the nonlinear elliptic equations for $\mathbf{d}$ and $\phi$ are unique minimizers of convex functionals. Hence, these numerical schemes are extremely efficient.

While we have considered only time discretizations here, the results can carry over to any consistent finite-dimensional Galerkin approximations (finite elements or spectral) since the proofs are all based on variational formulations with all test functions in the same space as the trial function.

To the authors' knowledge, these are the first schemes, for phase-field models of two-phase complex fluids, which decouple the computation of the director field 
function, phase function, velocity, and pressure and which lead to linear decoupled elliptic equations, in the stabilized approach, at each time step. We also presented ample numerical results to validate the model and the proposed schemes with qualitative comparisons with some benchmark experiments.

\section{REFERENCES}

[1] B. Akers And A. Belmonte, Impact dynamics of a solid sphere falling into a viscoelastic micellar fluid, J. Non-Newtonian Fluid Mech., 135 (2006), pp. 97-108.

[2] D. M. Anderson, G. B. McFadden, And A. A. Wheeler, Diffuse-interface methods in fluid mechanics, Annu. Rev. Fluid Mech., 30 (1998), pp. 139-165.

[3] P. P. Bhat, S. Appathurai, M. T. Harris, M. Pasquali, G. H. McKinley, and O. A. BASARAN, Formation of beads-on-a-string structures during break-up of viscoelastic filaments, Nature Phys., 6 (2010), pp. 625-631.

[4] B. R. Bird, C. F. Curtiss, R. C. Armstrong, and O. Hassager, Dynamics of Polymeric Liquids, Wiley, New York, 1994.

[5] F. Boyer and S. Minjeaud, Numerical schemes for a three component Cahn-Hilliard model, ESAIM Math. Model. Numer. Anal., 45 (2011), pp. 697-738.

[6] L. A. Caffarelli and N. E. Muler, An $L^{\infty}$ bound for solutions of the Cahn-Hilliard equation, Arch. Ration. Mech. Anal., 133 (1995), pp. 129-144.

[7] J. W. Cahn and J. E. Hilliard, Free energy of a nonuniform system. I. Interfacial free energy, J. Chem. Phys., 28 (1958), pp. 258-267.

[8] N. Condette, C. Melcher, And E. SÜli, Spectral approximation of pattern-forming nonlinear evolution equations with double-well potentials of quadratic growth, Math. Comp., 80 (2011), pp. 205-223.

[9] P.-G. De Gennes and J. Prost, The Physics of Liquid Crystals, Oxford University Press, New York, 1993.

[10] Q. DU, M. LI, AND C. LIU, Analysis of a phase field Navier-Stokes vesicle-fluid interaction model, Discrete Contin. Dyn. Syst. Ser. B, 8 (2007), pp. 539-556.

[11] C. M. Elliott and A. M. Stuart, The global dynamics of discrete semilinear parabolic equations, SIAM J. Numer. Anal., 30 (1993), pp. 1622-1663.

[12] J. L. Ericksen, Conservation laws for liquid crystals, Trans. Soc. Rheol., 5 (1961), pp. 23-34.

[13] J. L. ERICKSEN, Liquid crystals with variable degree of orientation, Arch. Rational Mech. Anal., 113 (1991), pp. 97-120.

[14] D. J. EYRE, Unconditionally gradient stable time marching the Cahn-Hilliard equation, In Computational and Mathematical Models of Microstructural Evolution, Mater. Res. Soc. Sympos. Proc. 529, 1998, MRS, Warrendale, PA, pp. 39-46.

[15] A. Fick, Poggendorff's annalen, J. Amer. Math. Soc. (1855), pp. 59-86.

[16] J. L. Guermond, P. Minev, And J. Shen, An overview of projection methods for incompressible flows, Comput. Methods Appl. Mech. Engrg., 195 (2006), pp. 6011-6045.

[17] M. E. Gurtin, D. Polignone, And J. Viñals, Two-phase binary fluids and immiscible fluids described by an order parameter, Math. Models Methods Appl. Sci., 6 (1996), pp. 815-831.

[18] J. HuA, P. Lin, C. Liu, ANd Q. WANG, Energy law preserving $C^{0}$ finite element schemes for phase field models in two-phase flow computations, J. of Comput. Phys., 230 (2011), pp. $7155-7131$.

[19] D. JACQmin, Calculation of two-phase Navier-Stokes flows using phase-field modeling, J. Comput. Phys., 155 (1999), pp. 96-127.

[20] B. Jerome, Surface effects and anchoring in liquid crystals, Rep. Progr. Phys., 54 (1991), p. 391.

[21] D. Kessler, R. H. Nochetto, And A. Schmidt, A posteriori error control for the AllenCahn problem: Circumventing Gronwall's inequality, M2AN Math. Model. Numer. Anal., 38 (2004), pp. 129-142.

[22] J. KIM, Phase-field models for multi-component fluid flows, Commun. Comput. Phys., 12 (2012), pp. 613-661.

[23] R. G. Larson, The Structure and Rheology of Complex Fluids, Oxford University Press, New York, 1999.

[24] F. M. LesLIE, Some constitutive equations for anisotropic fluids, Q. J. Mech. Appl. Math., 19 (1966), pp. 357-370.

[25] F. M. Leslie, Some constitutive equations for liquid crystals, Arch. Ration. Mech. Anal., 28 (1968), pp. 265-283. 
[26] F. H. Lin, On nematic liquid crystals with variable degree of orientation, Comm. Pure Appl. Math., 44 (1991), pp. 453-468.

[27] F. H. Lin, Mathematics theory of liquid crystals, in Applied Mathematics at the Turn of Century: Lecture Notes of the 1993 Summer School, Universidat Complutense de Madrid, 1995.

[28] F. H. Lin AND C. Liu, Nonparabolic dissipative systems modeling the flow of liquid crystals, Comm. Pure Appl. Math., 48 (1995), pp. 501-537.

[29] F. H. Lin And C. Liu, Static and dynamic theories of liquid crystals, J. Partial Differential Equations, 14 (2001), pp. 289-330.

[30] P. Lin AND C. LiU, Simulation of singularity dynamics in liquid crystal flows: A $C^{0}$ finite element approach, J. Comput. Phys., 215 (2006), pp. 348-362.

[31] P. Lin, C. Liu, and H. Zhang, An energy law preserving $C^{0}$ finite element scheme for simulating the kinematic effects in liquid crystal dynamics, J. Comput. Phys., 227 (2007), pp. 1411-1427.

[32] C. Liu And J. Shen, A phase field model for the mixture of two incompressible fluids and its approximation by a Fourier-spectral method, Phys. D, 179 (2003), pp. 211-228.

[33] C. Liu, J. Shen, And X. YAng, A new, thermodynamically consistent phase-field model and its numerical approximation for two-phase incompressible flows with different densities and viscosities, J. Sci. Comput., submitted.

[34] C. Liu and N. J. Walkington, An Eulerian description of fluids containing visco-hyperelastic particles, Arch. Ration. Mech. Anal., 159 (2001), pp. 229-252.

[35] J. M. Lopez And J. Shen, An efficient spectral-projection method for the Navier-Stokes equations in cylindrical geometries I. Axisymmetric cases, J. Comput. Phys., 139 (1998), pp. 308-326.

[36] J. Lowengrub and L. Truskinovsky, Quasi-incompressible Cahn-Hilliard fluids and topological transitions, R. Soc. Lond. Proc. Ser. A Math. Phys. Eng. Sci., 454 (1998), pp. 2617-2654.

[37] S. Minjeaud, An unconditionally stable uncoupled scheme for a triphasic CahnHilliard/Navier-Stokes model, Commun. Comput. Phys., 29 (2013), pp. 584-618.

[38] L. Rayleigh, On the theory of surface forces II, Philos. Mag., 33 (1892), pp. 209-220.

[39] J. Shen, Efficient spectral-Galerkin method I. Direct solvers for second- and fourth-order equations by using Legendre polynomials, SIAM J. Sci. Comput., 15 (1994), pp. 1489-1505.

[40] J. Shen, Efficient Chebyshev-Legendre Galerkin methods for elliptic problems, in Proceedings of ICOSAHOM'95, A. V. Ilin and R. Scott, eds., 1996, pp. 233-240.

[41] J. Shen AND X. YAnG, Energy stable schemes for Cahn-Hilliard phase-field model of two-phase incompressible flows, Chinese Ann. Math. Ser. B, 31 (2010), pp. 743-758.

[42] J. Shen And X. YAng, Numerical approximations of Allen-Cahn and Cahn-Hilliard equations, Discrete Contin. Dyn. Syst. Ser. A, 28 (2010), pp. 1169-1691.

[43] J. Shen And X. YAnG, A phase-field model and its numerical approximation for two-phase incompressible flows with different densities and viscositites, SIAM J. Sci. Comput., 32 (2010), pp. 1159-1179.

[44] J. SHEN, Modeling and numerical approximation of two-phase incompressible flows by a phasefield approach, in Multiscale Modeling and Analysis for Materials Simulation, W. Bao and Q. Du, eds., IMS Lecture Notes Monogr. Ser. 9, IMS, Institute of Mathematical Statistics, Hayward, CA, 2011, pp. 147-196.

[45] V. Tirtantmadja, G. H. McKinley, and J. J. Cooper-White, Drop formation and breakup of low viscosity elastic fluids: Effects of molecular weight and concentration, Phys. Fluids., 18 (2006), 043101.

[46] J. VAN DER WAALS, The thermodynamic theory of capillarity under the hypothesis of a continuous density variation, J. Statist. Phys., 20 (1893), pp. 197-244.

[47] X. Yang, J. J. Feng, C. Liu, and J. Shen, Numerical simulations of jet pinching-off and drop formation using an energetic variational phase-field method, J. Comput. Phys., 218 (2006), pp. 417-428.

[48] X. Yang, M. G. Forest, H. Li, C. Liu, J. Shen, Q. Wang, and F. Chen, Modeling and simulations of drop pinch-off from liquid crystal filaments and the leaky liquid crystal faucet immersed in viscous fluids, J. Comput. Phys., 236 (2013), pp. 1-14.

[49] P. Yue, J. J. Feng, C. Liu, and J. Shen, A diffuse-interface method for simulating two-phase flows of complex fluids, J. Fluid. Mech., 515 (2004), pp. 293-317.

[50] C. Zhou, P. Yue, J. J. Feng, C. Liu, and J. Shen, Heart-shaped bubbles rising in anisotropic liquids, Phys. Fluids, 19 (2007), 041703. 